\title{
Developmental and Functional Brain Impairment in Offspring from Preeclampsia-Like Rats
}

\author{
Xueyuan Liu • Wenlong Zhao • Haiyan Liu • \\ Yaoyue Kang • Chen Ye • Weirong Gu • Rong Hu • \\ Xiaotian Li
}

Received: 23 October 2014 / Accepted: 8 December 2014 / Published online: 10 January 2015

(C) The Author(s) 2015. This article is published with open access at Springerlink.com

\begin{abstract}
Preeclampsia is associated with developmental delay in infants and with an increased risk of various diseases in adulthood, including hypertension and epilepsy. It has been reported that several organs show developmental retardation and functional deficiency in offspring of preeclamptic rats. However, the developmental and functional changes in brains of the offspring of preeclamptic rats remain unknown. Here, we established a preeclampsialike rat model induced using $N \omega$-nitro-L-arginine methyl ester (L-NAME) to analyze the developmental and functional changes in brains of the offspring. Body and brain weights were decreased in the L-NAME group at postnatal day 0 (P0). However, there were no significant differences
\end{abstract}

Xueyuan Liu and Wenlong Zhao are the co-first authors.

Electronic supplementary material The online version of this article (doi:10.1007/s12035-014-9060-7) contains supplementary material, which is available to authorized users.

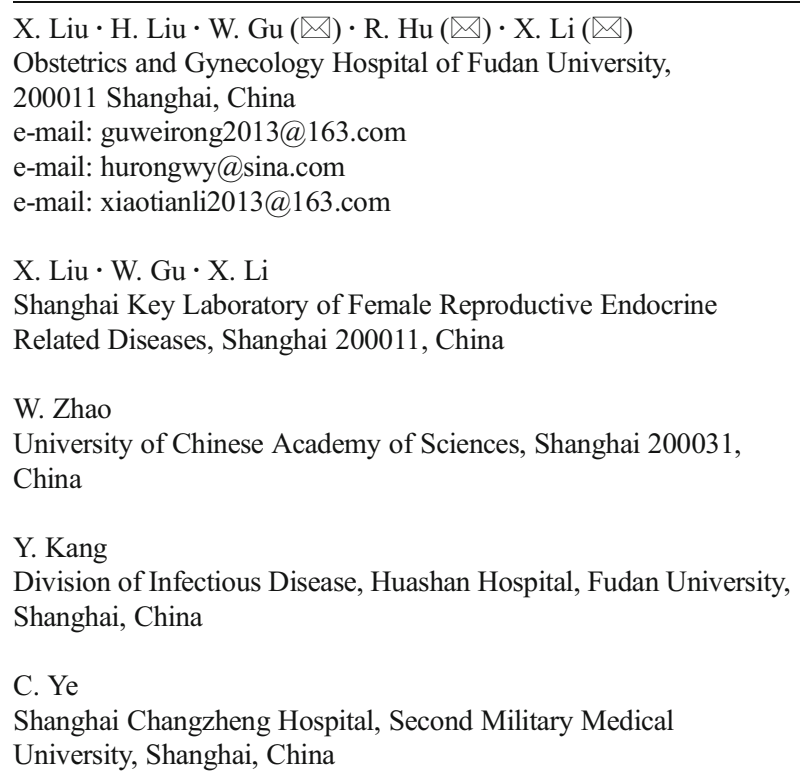

between the L-NAME and control groups in brain and body weights at P56. Upon further analysis, we detected a deficiency in neurogenesis, but not in apoptosis, which contributed to the smaller brains of the offspring in the LNAME group at P0. Additionally, we observed an increase in gliogenesis to compensate for the brain weights of the offspring at P56. Although the weight and laminar structure of the brains were essentially normal at P56, spatial learning and memory were severely impaired. We also found that adult hippocampal neurogenesis was disrupted in the offspring from preeclampsia-like rats, which may explain the cognitive deficiency. Moreover, qRT-PCR revealed a reduced expression of neurogenesis-related genes in the

X. Li

Institute of Biomedical Sciences, Fudan University, Shanghai 200011, China

\section{Li}

Shanghai Key Laboratory of Birth Defects, Shanghai 200011, China

W. Zhao

Institute of Neuroscience, Shanghai Institutes of Biological Sciences, Chinese Academy of Sciences, Shanghai 200031,

China 
offspring. Overall, we have described the deleterious effects of preeclampsia on the brains of offspring, providing clues to the cellular and molecular mechanisms involved in this pathogenesis, which may aid in the development of therapeutic approaches.

Keywords Preeclampsia-like · L-NAME · Water maze · Adult hippocampal neurogenesis · Gliogenesis

\section{Introduction}

Preeclampsia is associated with increased morbidity and mortality, and epidemiologic evidence has shown that infants from preeclamptic mothers are susceptible to respiratory distress syndrome, hypertension, stroke and/or epilepsy in adult life [1-3]. There are currently no effective strategies for preventing and treating preeclampsia, and little is known about the cellular and molecular mechanisms by which preeclampsia induces these adverse effects in offspring. Thus, it is necessary to establish preeclamptic animal models for further investigation of the adverse effects on the offspring of preeclamptic pregnancies.

Previous studies have reported that rats treated with $\mathrm{N \omega}$ nitro-L-arginine methyl ester (L-NAME), a nitric oxide synthase (NOS) inhibitor, during the last third of gestation exhibit preeclampsia-like symptoms $[4,5]$. Some studies have focused on the development and function of the lungs, kidneys, and vascular endothelium of infants from preeclamptic mothers or offspring from preeclampsia animal models [6-8]. Interestingly, the mid- to late stages of pregnancy (embryonic days 14.5-20.5 in rodents; weeks 20-40 in humans), which is the timeframe in which the onset of preeclampsia occurs (approximately 20 weeks), are critical for fetal brain development in utero [9-11]. However, there are only a few scattered studies concerning the developmental and functional changes in the nervous systems of offspring from preeclampsia pregnancies in humans and rats $[12,13]$. To our knowledge, the brain development and function of the offspring of preeclamptic rats have not been previously documented. Therefore, the aims of this study were to investigate whether preeclampsia disrupts the development and function of the brain and to explore the mechanisms underlying these perturbations in the offspring of preeclamptic dams.

\section{Materials and Methods}

For detailed description of the material and methods please refer to the online-only Data Supplement.
Animals and General Body Parameter Measurements

Adult pregnant Sprague-Dawley rats were purchased from SLAC Laboratory Animal Co. Ltd. and housed individually. All animals were given free access to food and water. Following birth, the pups were kept in the cages with their mothers. All pups were weighed with an electric scale. All procedures were approved by the Animal Care and Use Committee of the University of Fudan.

\section{Establishment of the Preeclampsia-Like Rat Model}

The pregnant dams were randomly divided into two groups, which were given $50 \mathrm{mg} \mathrm{kg}^{-1}$ day $^{-1}$ of L-NAME or pure water via daily gavage from day 14.5 to day 20.5 of gestation [6]. Their systolic blood pressure (SBP) and urokinase protein levels were detected with a BP-2000 Blood Pressure analyzer (BP-2000, Visitech Systems Inc., North Carolina, USA) and a Bayer ADVIA 1650 analyzer (ADVIA 1650, Bayer, Leverkusen, Germany), respectively.

\section{Water Maze Test}

We used 15 P56 male rats from the L-NAME treatment group and 15 P56 rats from the control group in the water maze test. And it was carried out as described previously [14] but with modification as described in the Data Supplement.

Immunohistochemistry

The $\mathrm{P} 0$ rat brains were sliced into $20-\mu \mathrm{m}$ coronal sections on glass slides, while the P56 rat brains were sliced to $30-\mu \mathrm{m}$ free-floating coronal sections. And images were acquired with a SP8 confocal microscope (Leica Microsystems, Wetzlar, Germany). The information about the primary and secondary antibodies and the applied working concentrations are provided in Supplemental Table 1.

\section{Hematoxylin and Eosin Staining}

The slices were treated with a hematoxylin and eosin staining kit (Beyotime, C0105, Wuhan, China) following the manufacturer's instructions.

\section{Quantitative Real-Time PCR Assays}

Total cortical RNA from P0 rats or the hippocampus of P56 rats was subjected to extraction using TRIzol (Invitrogen, 15596026, Carlsbad, CA, USA). Reverse transcription and first-strand cDNA synthesis were performed using the PrimeScript ${ }^{\mathrm{TM}}$ RT reagent kit (TaKaRa, RR047A, Japan). Quantitative real-time PCR was carried out with the EvaGreen dye (Biotium, catalog \# 31000, USA). The primers used in the 
qRT-PCR assays were specific for neurogenesis-associated genes. The primer sequences are listed in Supplemental Table 2.

\section{Statistical Analyses}

Statistical calculations were conducted using GraphPad Prism 5 software. In the statistical graphs, the error bars represent the s.e.m. Statistical significance was determined using Student's $t$ test, the Mann-Whitney test, or one-way ANOVA, and $P$ values $<0.05$ were considered statistically significant.

\section{Results}

Establishment of the Preeclampsia-Like Rat Model

To detect the consequences of preeclampsia in the brains of rat offspring, we first examined the perinatal effects of treating pregnant rats with L-NAME to confirm whether we had successfully established a preeclampsia-like animal model. We found that L-NAME administration resulted in increased blood pressure levels associated with proteinuria in the pregnant rats (Fig. 1a, b). Then, we obtained 84 newborn rat pups from the control group and 56 from the L-NAME group. We found that in the L-NAME group, the fetal mortality rate was increased (Fig. 1c), and two pups exhibited hindlimb necrosis (Fig. 1d). Excluding the dead pups and those with hindlimb necrosis from further analyses, the body weight at birth was significantly lower in the L-NAME group [5.332 $\pm 1.186 \mathrm{~g}]$ than in the control group [7.503 $\pm 1.341 \mathrm{~g}] ; p<0.001$ (Fig. 1e). However, there were no significant differences in body weight between the two groups from postnatal day 7 onward (Fig. 1e). These results were consistent with previous reports [6] and suggested that our preeclampsia-like animals were suitable for further analysis of the developmental and functional changes in the brains of offspring.

Evaluation of Brain Development in the Offspring of Pregnant Rats Treated with L-NAME

We found that the offspring in the L-NAME group exhibited smaller brains at P0, but they were not significantly different at P56 compared with the control group (Fig. 2a, b). Furthermore, the ratio of the brain weight to body weight was
Fig. 1 Establishment of a preeclampsia-like model in rats. a Systolic blood pressure of pregnant rats in the L-NAME and control groups. The mean \pm s.e.m. is shown. Statistical analysis was performed using Student's $t$ test. b Urokinase protein levels in pregnant rats in the L-NAME and control groups. The mean \pm s.e.m is shown. Statistical analysis was performed with the MannWhitney test. c Number of rat pups per litter in the L-NAME and control groups. The mean \pm s.e.m. is shown. Statistical analysis was performed with the MannWhitney test. d Characteristic gross anatomic pathologic findings in $\mathrm{P} 0$ rats from preeclampsia-like mothers compared with control mothers. Arrows indicate limb defects. e Body weight at $\mathrm{P} 0,7,16$, and 56 of rat pups in the L-NAME and control groups. The mean \pm s.e.m. is shown. Statistical analysis was performed with the MannWhitney test. $* * P<0.01$; $* * * P<0.001$ a

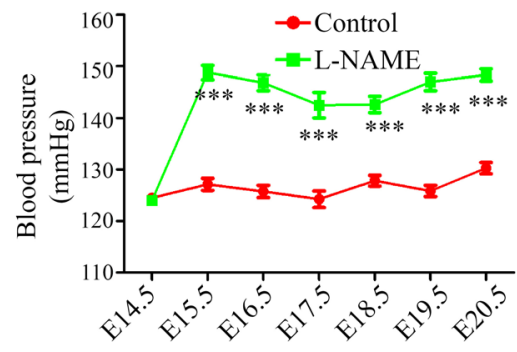

C

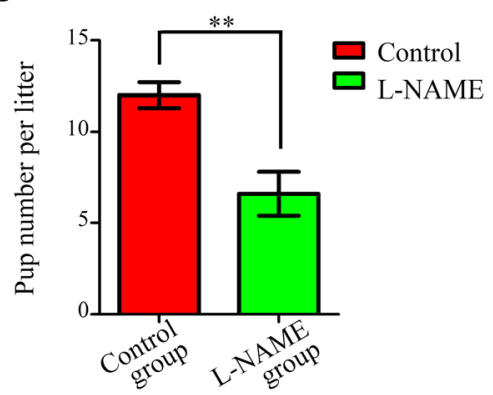

e

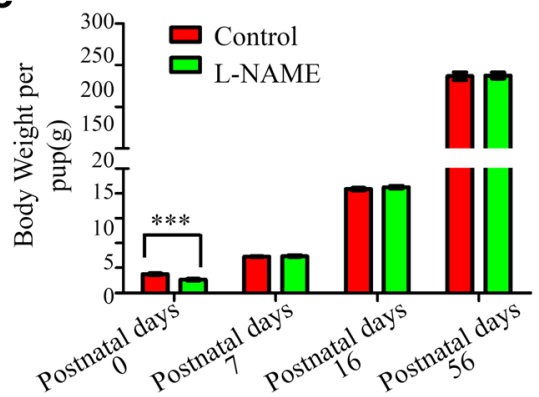

b

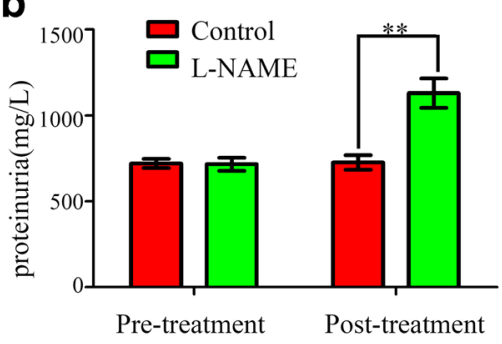

d

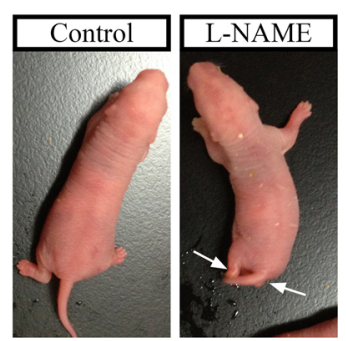


Fig. 2 Evaluation of brain development in offspring from the L-NAME group. a Dorsal view of P0 (left panel) and P56 (right panel) rat brains in the $\mathrm{L}$ NAME and control groups. b Brain weight at $\mathrm{P} 0$ and $\mathrm{P} 56$ in the L-NAME and control groups. The mean \pm s.e.m is shown. Statistical analysis was performed with the Mann-Whitney test. c Ratio of brain weight to body weight at $\mathrm{P} 0$ and P56 in the L-NAME and control groups. d Coronal sections of $\mathrm{P} 0$ (upper panel) and P56 (lower panel) rat brains stained with hematoxylin and eosin. e Statistical results for cortex thickness in P0 and P56 rat pups in the L-NAME and control groups. The mean \pm s.e.m. is shown. Statistical analysis was performed using Student's $t$ test. $* * P<0.01$

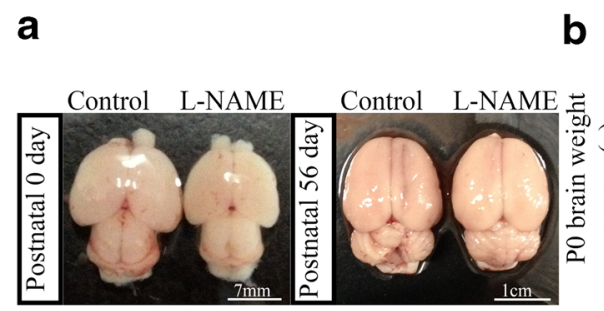

b

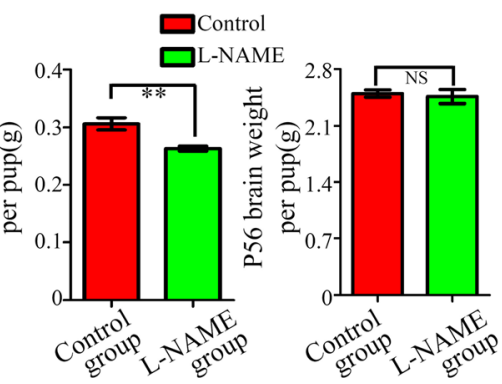

C

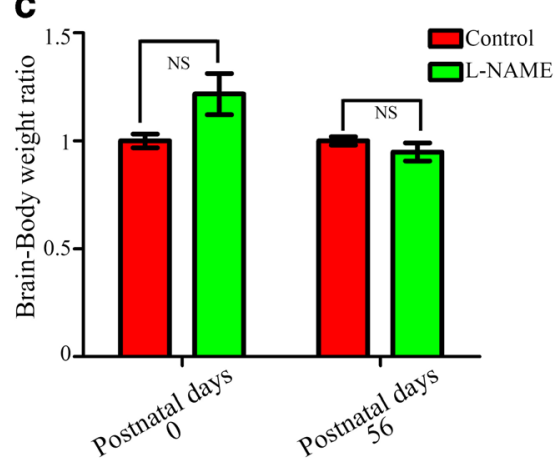

d
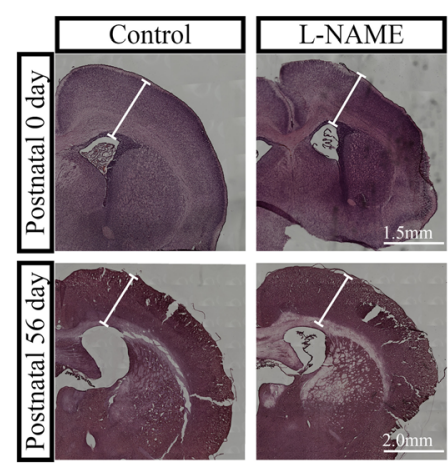

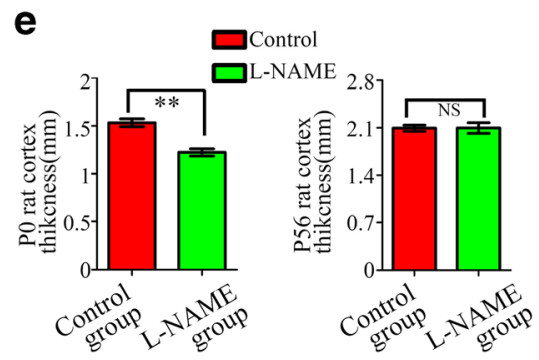

unchanged in the L-NAME group (Fig. 2c). Hematoxylin and eosin staining showed that the radial dimension (thickness) was decreased at P0, but not at P56, in the L-NAME group compared with the controls (Fig. 2d, e). These results indicated that the growth of the whole body, including the brain, is delayed prenatally, but this developmental retardation could recover after delivery.

To elucidate the cellular and molecular mechanisms underlying the defects in early brain development observed in the L-NAME group offspring, we evaluated proliferation and apoptosis in $\mathrm{P} 0$ rats through immunostaining for $\mathrm{PH} 3$ and activated cleavage caspase 3 , respectively. Acute BrdU incorporation assays were also performed to examine cell proliferation. We evaluated the numbers of $\mathrm{PH}^{+}$cells as well as $\mathrm{BrdU}^{+}$and activated cleavage caspase $3^{+}$cells at P0 within the ventricular zone (VZ) of the neocortex. Interestingly, we observed a decrease in the numbers of $\mathrm{PH}^{+}$and $\mathrm{BrdU}^{+}$cells in the neocortex in the L-NAME group (Fig. 3a-d). However, the number of activated cleavage caspase $3^{+}$cells was not significantly different between the two groups (Fig. 3e, f). To investigate the mechanism at the molecular level, we examined the expression levels of several genes involved in the proliferation of radial glia, including Fgf2 and others listed in Supplemental Table 2 [15]. Using RT-PCR, we detected reduced expression of the Fgf2, Creb, and Ep300 genes in the L-NAME group compared with the control group (Fig. 3g).

We next questioned whether L-NAME treatment affected the number and structure of radial glia, which are recognized as neural stem cells and provide the scaffold for the migration of newborn neurons. We analyzed the morphological changes in the radial glial scaffold and the differentiation of neural progenitor cells via immunostaining for the cell-type-specific markers Nestin and Tuj1, respectively. We found that the morphology of the radial glia scaffold in the L-NAME group was similar to that in the control group. However, we discovered that the Nestin ${ }^{+}$regions and $\mathrm{Tuj}^{+}$regions in the L-NAME group were thinner than in the control group (Fig. 4a-c). Therefore, we concluded that the lower brain weights and smaller brain sizes may have resulted from a deficiency in the proliferation of neural progenitor cells in early developmental stages, without affecting the differentiation of neural progenitor cells or the morphology of the radial glial scaffold. 
Fig. 3 Analysis of progenitor cell proliferation and apoptosis in newborn offspring in the LNAME and control groups. a $\mathrm{PH} 3$ immunofluorescence in coronal sections of the neocortex. b BrdU immunofluorescence in coronal sections of the neocortex after $4 \mathrm{~h}$ of BrdU incorporation. c Number of PH3-positive cells in the VZ per section. $\mathbf{d}$ Number of BrdU-positive cells in the VZ per section. e Immunofluorescence staining of coronal sections of the neocortex with an antibody against activated cleavage caspase3. f Number of activated cleavage caspase3-positive cells per section. $\mathbf{g}$ mRNA levels of neurogenesis-related genes at $\mathrm{P} 0$ rat offspring from the L-NAME and control groups, as detected via qRT-PCR. $V Z$ ventricular zone, $S V Z$ subventricular zone. $N>3 / 3$, the number of sections examined from at least three rats each group. The mean \pm s.e.m. is shown. Statistical analysis was performed using Student's $t$ test. $* * * P<0.001$
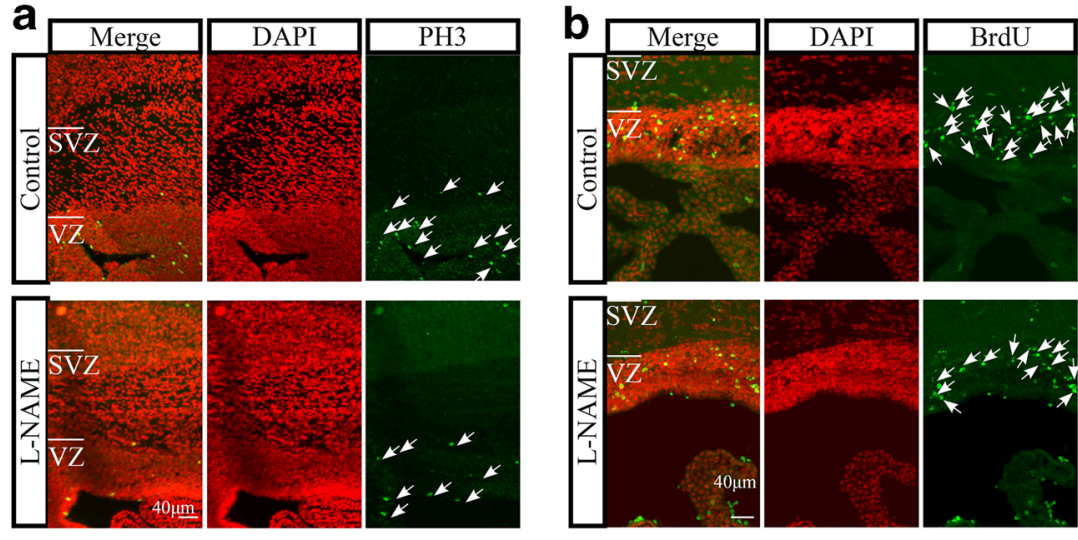

C

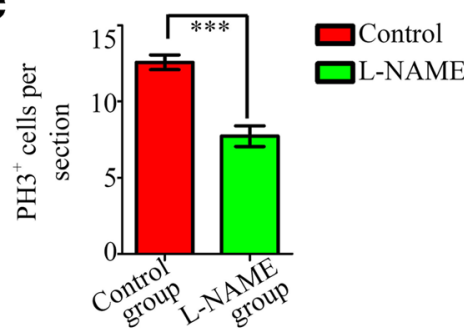

e

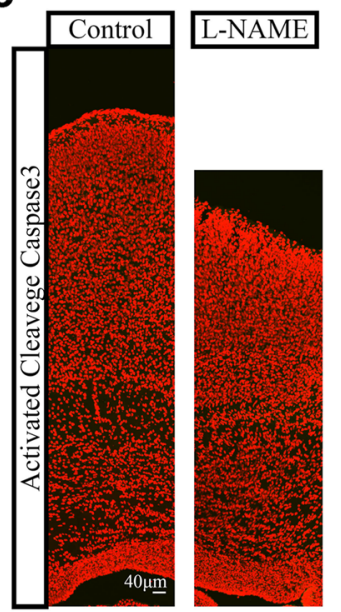

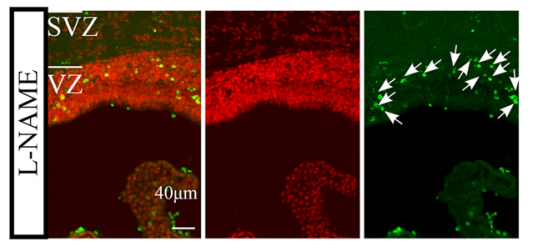

d

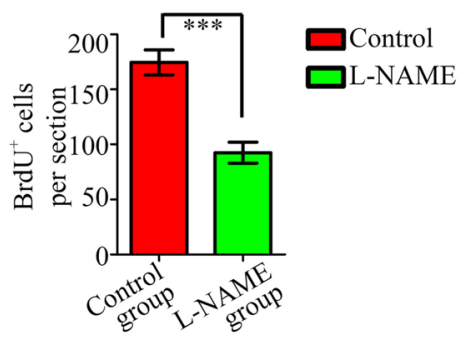

f

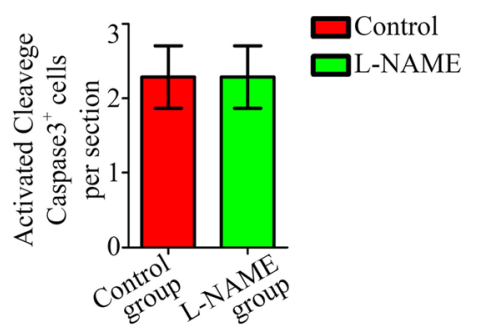

$\mathbf{g}$

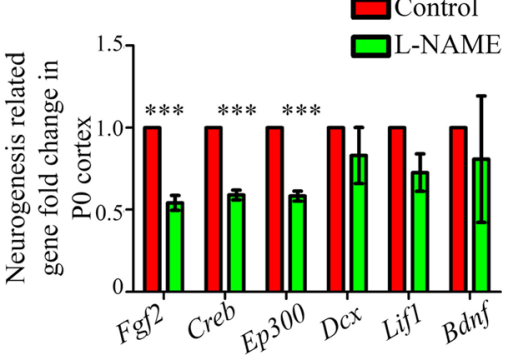

Next, we investigated the cellular mechanism underlying the recovery observed in adult brains in the L-NAME group. First, we examined the laminar structure of adult brains via immunostaining for Ctip2 and Tbr1, which are deeper layer and thinner layer markers, respectively. We found that the patterns of the $\mathrm{Ctip}^{+}$and $\mathrm{Tbr}^{+}$cell layers were similar between the two groups (Fig. S1a, b).

Glial cells constitute nearly $50 \%$ of the cells in the mammalian brain, and astrocytes, which proliferate postnatally, are the largest glial population [16]. Thus, we explored the numbers of neurons and astrocytes in adult brains through immunostaining for the neuronal marker NeuN and the astrocytic marker GFAP. Intriguingly, we found that the number of
$\mathrm{GFAP}^{+}$cells was increased in the whole adult brains from the L-NAME group following reduction of the number of $\mathrm{NeuN}^{+}$cells (Fig. 5a-d). These data suggested that the proliferation of astrocytes may be a contributing factor in the recovery of brain weight among the offspring of preeclamptic dams.

Impairment of Learning and Memory in the Offspring of the L-NAME Group

Although the brain weight and laminar structure were essentially normal in the offspring of the preeclampsia-like rats at adulthood, we investigated the existence of possible 
Fig. 4 The radial glial scaffold of newborn offspring in the LNAME and control groups. a Immunofluorescence images of Nestin and Tuj1 in coronal sections of the neocortex. b Thickness of the Nestinpositive region at $\mathrm{P} 0$ rat offspring from the L-NAME and control groups. c Thickness of the Tuj1positive region at $\mathrm{P} 0$ rat offspring from the L-NAME and control groups. $V Z$ ventricular zone, $S V Z$ subventricular zone, $I Z$ intermediate zone, $C P$ cortical plate. $N>3 / 3$, the number of sections examined from at least three rats each group. The mean \pm s.e.m. is shown. Statistical analysis was performed using Student's $t$ test. ${ }^{* *} P<0.01$
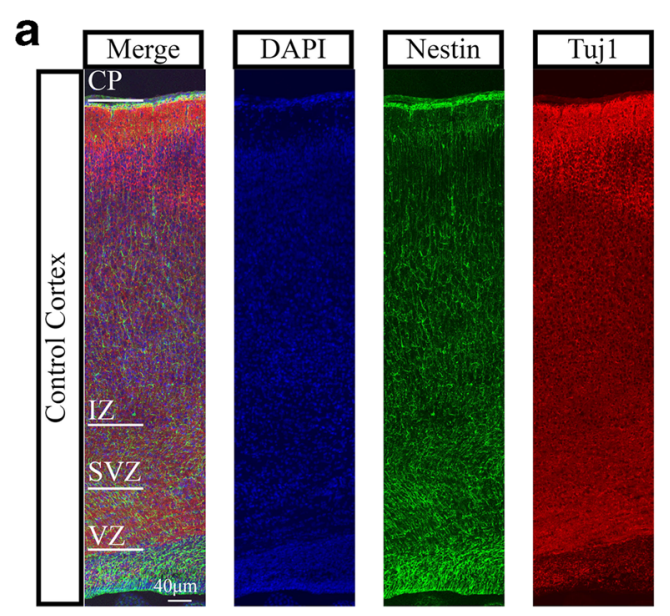

\section{b}
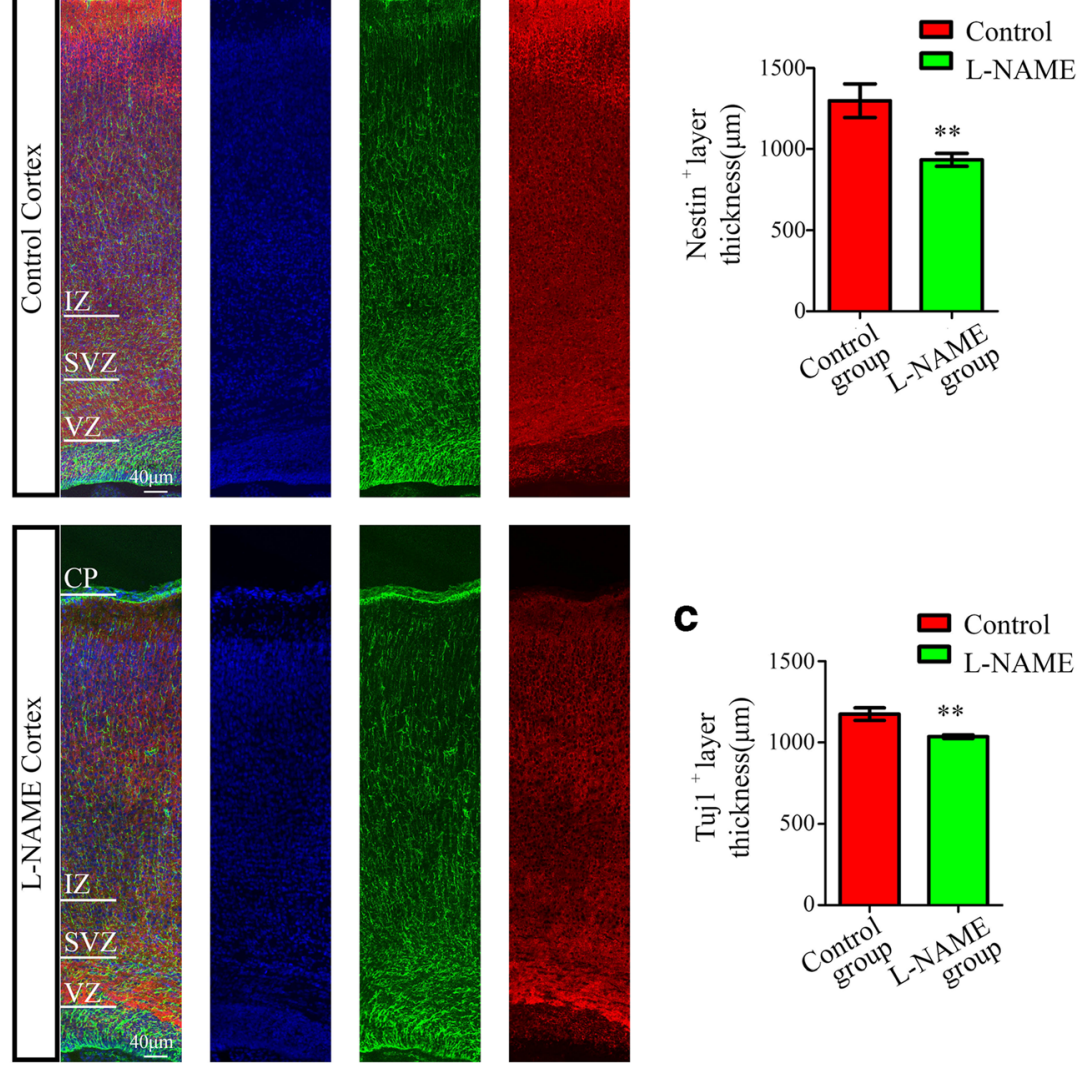

functional impairment. We selected P56 male offspring from the control and L-NAME groups to analyze their learning and memory abilities using the water maze test. During the training stage, the results revealed that the rat offspring from the control group progressively learned the task from session 1 to session 9, whereas the rat offspring from the L-NAME group were markedly slower and less efficient at learning the task (Fig. 6a). However, no significant differences in swimming speed were found between the two groups (Fig. 6b). Thus, the spatial learning abilities of rat offspring from preeclamptic pregnancies are impaired.

At the probe stage, the results revealed that the rat offspring from the control group spent more time in the target quadrant and less time in the target contra-quadrant than the offspring from the L-NAME group (Fig. 7a). And offspring from the LNAME group displayed significantly higher latencies to escape to the training platform area compared with the control group (Fig. 7a). Additionally, we found that the frequency of platform crossing in the control group was higher than that in the L-NAME group (Fig. 7e, f). Higher percentages of time and a greater swimming distance within the target quadrant were observed in the control group than in the L-NAME group (Fig. $7 b, c)$. The recorded swimming speeds, including the mean speed, maximum speed, and the speeds in the four quadrants, did not show differences between the two groups (Fig. 7d). Therefore, we have demonstrated that the rat offspring from preeclamptic pregnancies display deficiencies in spatial learning and memory.

Impairment of Adult Hippocampal Neurogenesis in Offspring from the L-NAME Group

To reveal the cellular and molecular mechanisms underlying the functional impairment of brain function in the offspring of preeclamptic rats, we examined adult hippocampal neurogenesis, which is associated with the disruption of spatial learning and memory abilities [17]. Thus, the rat offspring were injected with BrdU solution, as shown in a schematic diagram (Fig. 8a). We found that the numbers of $\mathrm{BrdU}^{+}$cells in the adult hippocampus were dramatically decreased in the L-NAME group (Fig. 8b, c). Next, we used RT-PCR to detect the expression levels of genes associated with adult hippocampal neurogenesis [15]. We observed under-expression of the $F g f 2$ gene in the L-NAME group (Fig. 8d). This defect in adult hippocampal neurogenesis may be the cellular and molecular mechanism underlying the deficiency in spatial learning and memory in offspring from the L-NAME group. 

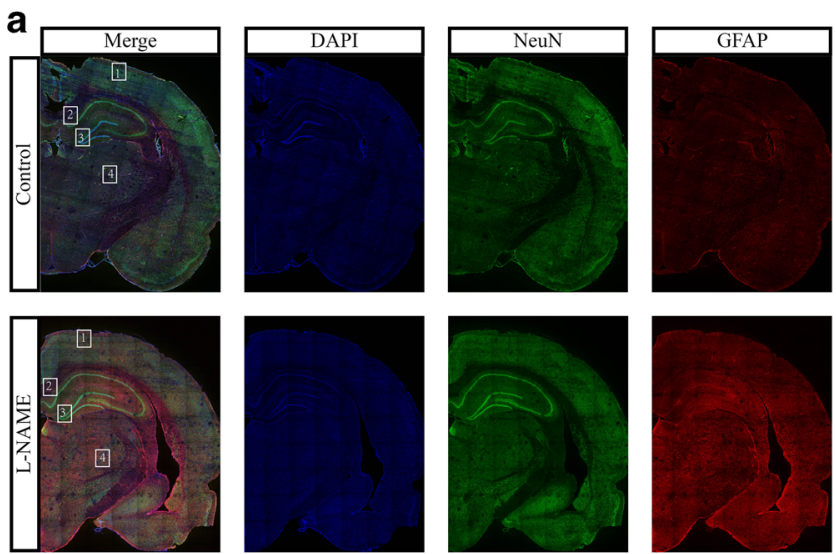

b
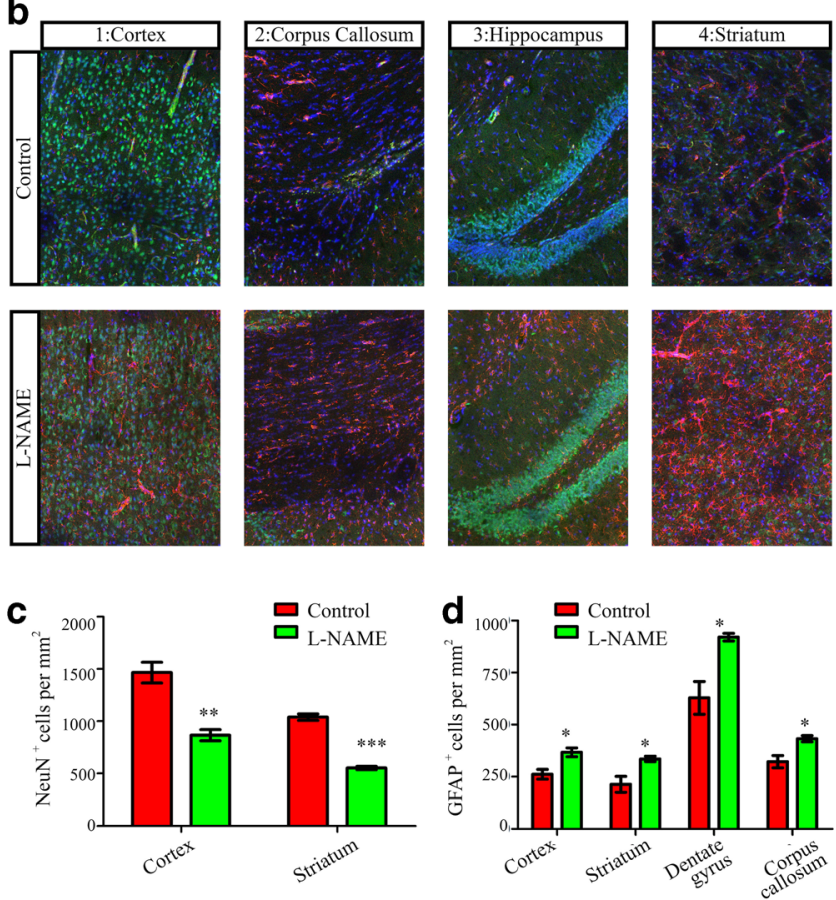

Fig.5 Quantification of neurons and astrocytes in P56 offspring from the L-NAME and control groups. a NeuN and GFAP immunofluorescence in coronal sections of the neocortex from the L-NAME and control groups. b Enlarged images from regions 1, 2, 3, and 4 in a. Region 1, cortex; region 2, corpus callosum; region 3, hippocampal DG; region 4, striatum. c The number of NeuN-positive cells in the neocortex and striatum per section in rat offspring from the L-NAME and control groups. $\mathbf{d}$ The number of GFAP-positive cells in the neocortex, striatum, dentate gyrus, and corpus callosum per section in rat offspring from the L-NAME and control groups. $N>3 / 3$, the number of sections from at least three rats of each group. The mean \pm s.e.m. is shown. Statistical analysis was performed using Student's $t$ test. $* P<0.05, * * P<0.01, * * * P<0.001$

\section{Discussion}

In the present study, we confirmed that treatment of rats with the NOS inhibitor L-NAME from E14.5 to birth, which is a critical stage for brain development, could cause a preeclampsia-like syndrome and IUGR in the offspring. At birth, body weight was lower in the L-NAME group than in the control group, and the L-NAME group showed a significant delay in brain development. L-NAME administration may have interfered with brain development simply by impairing maternofetal exchange, thereby reducing the nutrition available to the offspring, since the brain is a fetal organ with the highest metabolism, consuming the largest amount of nutrients during development. And in previous studies, poor nutrition has been shown to result in poor neurodevelopment by impairing neurogenesis or other cellular processes [18]. In our rat model, we observed a disruption of neurogenesis in the offspring from the L-NAME group. Moreover, growth factors are crucial for translating nutritional fuel into brain growth through a series of signaling pathway cascades [19]. The production of growth factors can be inhibited by a lack of nutrients [20]. Interestingly, in offspring from the L-NAME group at P0, we detected a reduced expression of $\mathrm{Creb}$, $E p 300$, and $F g f 2$, which are important for brain growth and neurogenesis. Therefore, increasing nutrient supplementation or providing some specific critical nutrition component may be beneficial for the in utero brain development of offspring of preeclamptic mothers.

Interestingly, we found that the low body weight and brain weight were recovered approximately 1 week after birth. The results concerning body weight were consistent with previous data. The observed rapid growth that abolished the differences in brain size were similar to the findings described in a previous report addressing lung development in rats showing decreases in lung volume at $\mathrm{P} 0$ that were resolved within the first 2 postnatal weeks [6]. These results are also consistent with observations from human cases. Babies from preeclamptic pregnancies show a reduced head circumference and a low body weight at age 2 years [21]. There are some reports showing rapid recovery in body weight or head circumference in children or adult humans, which may be due to sufficient nutrient uptake after birth. Thus, we propose that the fetus might receive cues from the mother's preeclamptic state and transiently alter its metabolism, hormone production, tissue sensitivity to circulating factors, and even the expression of certain genes to adapt to the mother's health while in utero. After delivery, the development of pups is partially restored to normal without these cues from mothers, which might be the reason for the rapid recovery observed in the body weight and development of organs.

It is unfortunate that the unique developmental pattern of the brain may not completely recover. First, embryonic brain development is critical for the neurodevelopment of vertebrates throughout life. In rodents, several pivotal neurodevelopment processes occur between E14 and E20 parallel to the onset of preeclampsia in humans. And the developmental pattern is similar in humans. These processes include embryonic neurogenesis, neuronal migration, neuronal morphogenesis, and gliogenesis which produces minority of glia and/or glial precursors $[9,22]$. Postnatally, the major 
a

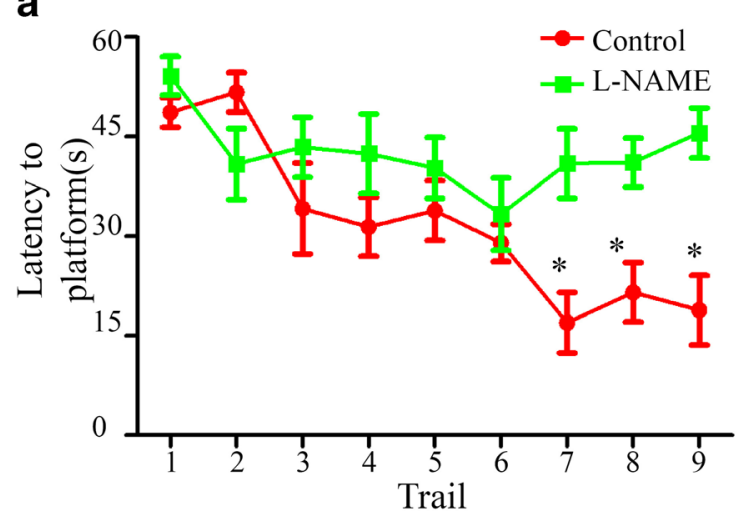

Fig. 6 Spatial learning is impaired in offspring from preeclamptic pregnancies. a Latency to escape onto the hidden platform in rat offspring from the L-NAME and control groups during training trials. b Mean speed during the total training time $(1 \mathrm{~min})$ in rat offspring from the L-NAME

neurodevelopment processes in both rodents and humans are gliogenesis and experience-dependent synapse remodeling/ pruning $[23,10]$. Although the offspring have separated from

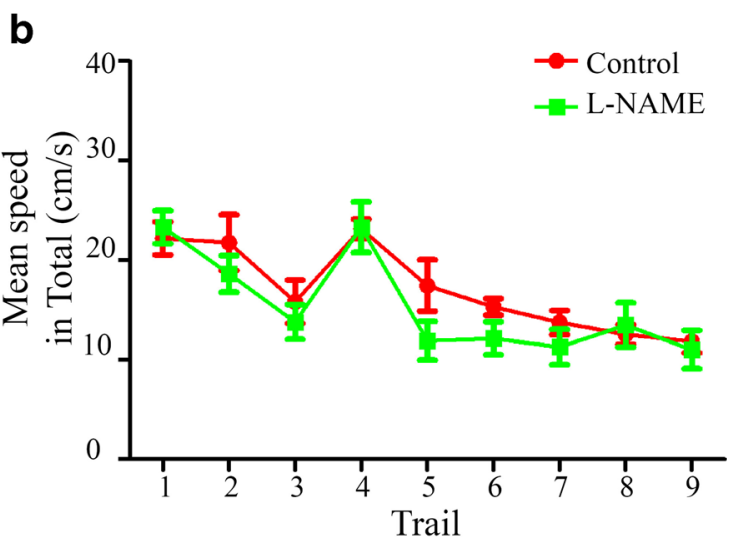

and control groups. The mean \pm s.e.m. is shown. Statistical analysis was performed using Student's $t$ test. $* P<0.05$. $N=15 / 15$, number of rats tested in training trials from the control and L-NAME groups, respectively

the mother at this stage and may obtain appropriate nutrition, certain biological events such as neurogenesis, which predominantly occurs during the embryonic stage, cannot be repaired a

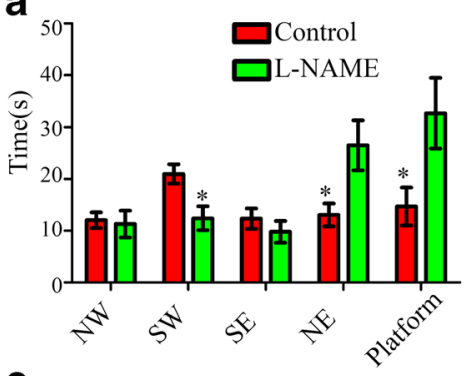

c

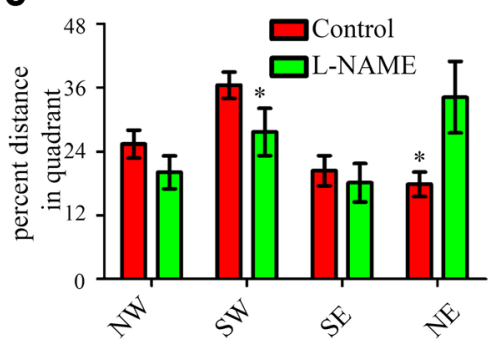

b

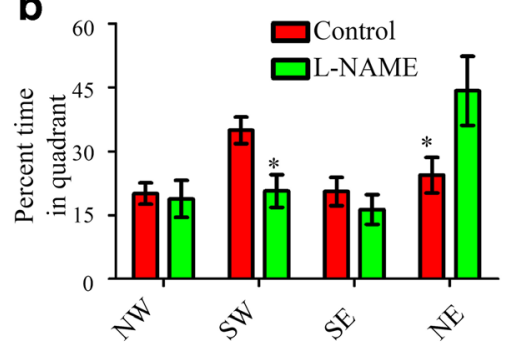

d

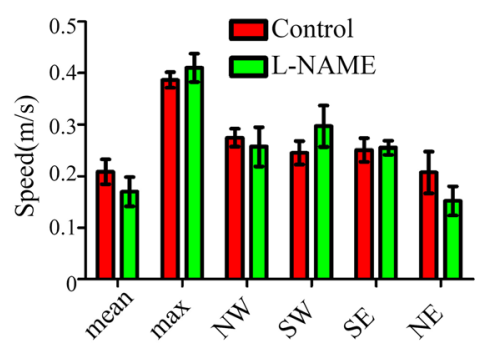

e

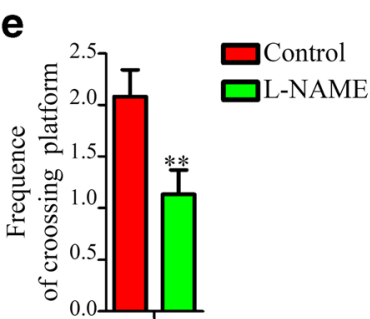

f
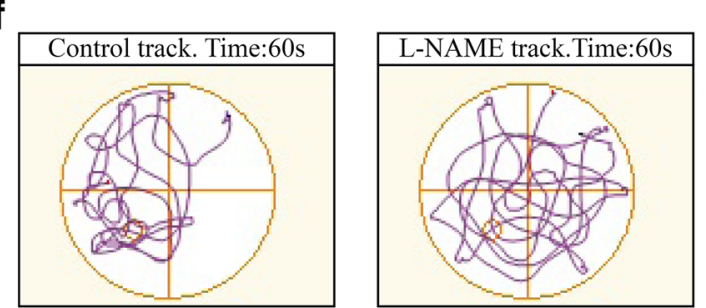

Fig. 7 Spatial memory is impaired in offspring from preeclamptic pregnancies. a Time spent in each quadrant and latency to escape onto the training platform area of the Morris water maze in the probe trial in the LNAME and control groups. b Percentage of time spent in each quadrant of the Morris water maze during the probe trial in the L-NAME and control groups. c Percentage of the distance swum in each quadrant of the Morris water maze during the probe trial in the L-NAME and control groups. d
Mean and maximum speeds in the total probe time as well as the mean speed in each quadrant in the L-NAME and control groups. e Frequency of crossing over the platform area in the L-NAME and control groups in probe trial. f Typical behavior tracks of the tested rat offspring in the L-NAME and control groups. The mean \pm s.e.m. is shown. Statistical analysis was performed using Student's $t$ test. $* P<0.05$; $* * P<0.01 . N=15 / 15$, numbers of rats in the test trials from the control and L-NAME groups, respectively 
Fig. 8 Adult hippocampal neurogenesis is impaired in offspring from preeclamptic pregnancies. a Scheme of BrdU solution administration in offspring in the L-NAME and control groups. b Typical cases showing BrdU-labeled cells in the adult hippocampus in the LNAME and control groups following immunofluorescence staining with a BrdU antibody (green) and staining with DAPI (red). Arrows indicate BrdUpositive cells. c Total BrdU per $\mathrm{mm}^{3}$ in the adult hippocampus in the offspring of L-NAME and control rats. d Expression levels of neurogenesis-related genes in the adult offspring of L-NAME and control rats, as detected via qRT-PCR. The mean \pm s.e.m. is shown. Statistical analysis was performed with Student's $t$ test. $* * P<0.01, * * * P<0.001$. NS, $P>0.05 . N=3 / 3$, numbers of rats examined in these assays from each group a

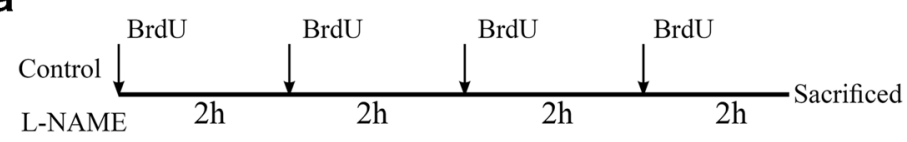

b

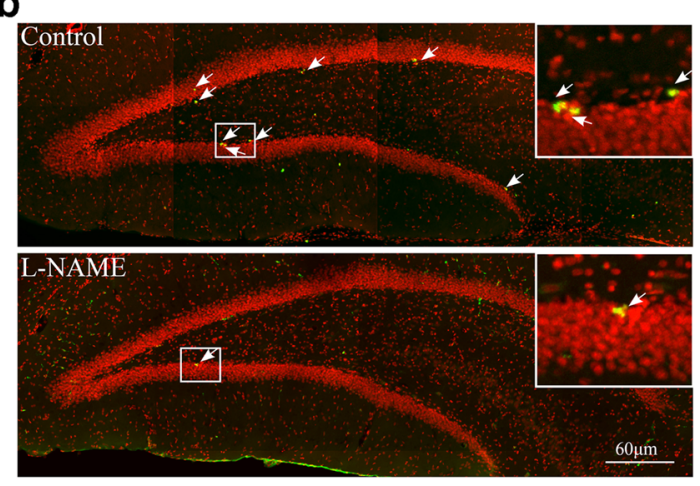

C

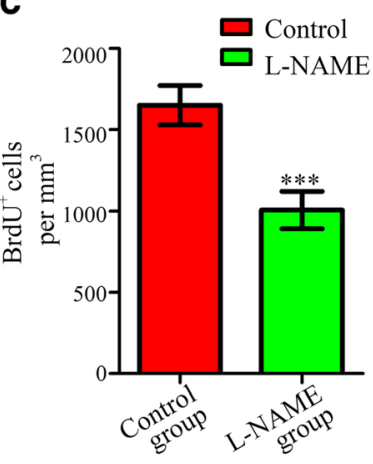

d

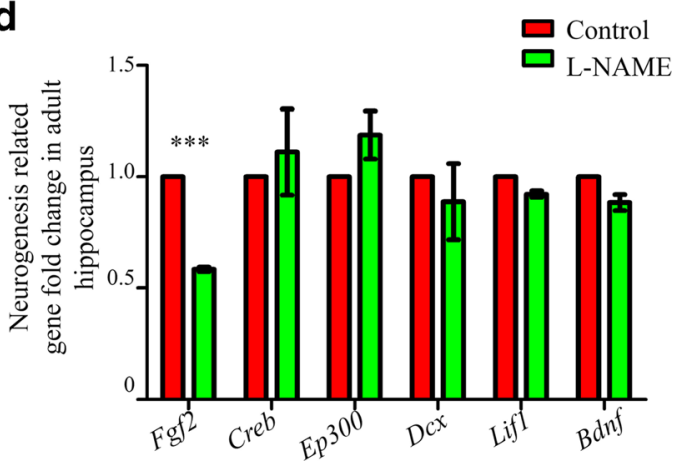

at postnatal stage. In fact, we observed overproduction of astrocytes, which may contribute to the recovery of brain weight and size, and the numbers of neurons in two brain regions in the offspring of preeclampsia-like rats remained lower than in normal brains.

In addition, extensive studies have shown that the functions of many organs or tissues, such as vascular and renal endothelia, are impaired or dysfunctional in the offspring of mothers with preeclampsia or other diseases, showing no resolution through sufficient nutrition or other supplementation after birth $[24,8,25]$. In the present study, the rats from the preeclampsia-like group exhibited defects in spatial learning and memory, although no macroscopic defects were observed in the brains of the adult offspring from this group. In humans, one paper has reported that the hypertensive diseases of pregnancy, including gestational hypertension and preeclampsia, are associated with neurocognitive outcomes in middle childhood. The authors of this work found that scores on the Peabody Picture Vocabulary Test-Revised (PPVT-R) are lower in offspring from pregnancies with maternal hypertension than in children from normotensive pregnancies [12].

Furthermore, we observed a deficiency in neurogenesis in the adult hippocampus, which may lead to the defective spatial learning and memory detected in the L-NAME group. Over the last decade, many studies have shown that new neurons can be continually generated in the subventricular zone of the lateral ventricles (SVZ) and in the subgranular zone of the hippocampal dentate gyrus (SGZ). The newborn neurons from the SVZ migrate into the olfactory bulb, which contributes to olfactory sensation in mammals [26]. The newborn neurons in the SGZ of the dentate gyrus incorporate into the molecular layer and integrate into neuronal circuits involved in learning and memory and in other cognitive processes $[27,28]$. The deficiency in adult hippocampal neurogenesis may cause the defects in spatial learning and memory observed in rat offspring from preeclampsia-like pregnancies. Alternatively, astrocytes are crucial to the regulation of synaptic connectivity and function. Therefore, we do not rule out a contribution of the rapid and robust generation of astrocytes in the DG region to the defective spatial learning and memory detected in the water maze test.

However, the mechanisms underlying these long-term effects in offspring following L-NAME-induced preeclampsia remain unknown. We propose two possible explanations for these consequences. 
First, accumulated evidence has indicated that adult neural stem cells, including adult hippocampal neural stem cells, are derived from radial glia in the embryo [29, 30]. Additionally, we observed that the offspring from the L-NAME group rats show deficiencies in cell proliferation in the $\mathrm{VZ}$ at $\mathrm{P} 0$ and in neurogenesis in the adult hippocampus. Thus, we speculate that the number of embryonic radial glia, which would develop into adult stem cells in the hippocampus, is reduced after L-NAME administration.

Second, genes participating in the proliferation of adult stem cells are downregulated in offspring from preeclampsia-like rat. We observed that the mRNA levels of genes involved in proliferation, such as Fgf2, Creb, and Ep300, were decreased in P0 cortex and/or adult hippocampus in the offspring from the L-NAME group [31, 32]. Fgf2 belongs to the family of fibroblast growth factors, which are known to play critical roles in cell proliferation and differentiation. $\mathrm{Fg} f 2^{-/-}$mice have been shown to present an approximately $10 \%$ reduction in cerebral cortex size and neuronal density, and these mice show impairments in memory at adulthood [33-35]. Furthermore, these mice exhibited defects in spatial learning and memory. Interestingly, it has been reported that $F g f$, $\mathrm{Creb}$, and Ep300 are involved in pathogenesis of epilepsy which is susceptive in offspring from preeclamptic mothers [36-38]. And localized overexpression of $F g f 2$ could reduce epileptogenesis in an epilepsy rat model. Therefore, the changes in expression levels of the above genes may contribute to the deficiencies in adult hippocampal neurogenesis and in cell proliferation observed in $\mathrm{P0}$ rats following the administration of L-NAME.

Moreover, the expression levels of $\mathrm{Fgf} 2, \mathrm{Creb}$, and other genes are known to be dynamically regulated during development and in response to environmental cues. It has been reported that the expression of $F g f 2$ can be regulated by modifying the methylation status within gene promoters [39, 40]. However, we did not detect any difference in methylation status within the $\mathrm{Fgf2}$, Creb, and Ep300 promoters between the control and L-NAME groups (data not shown). Further analysis is necessary to reveal the underlying mechanism in future works.

Taken together, we found perturbations in neurogenesis and in spatial learning and memory as well as gliosis in offspring from preeclampsia-like rats. The deficiencies in adult hippocampal neurogenesis, with under-expression of related genes, and gliosis provide insight into the mechanisms causing the deficiencies in spatial learning and memory. The combined results of our study may provide a plausible model for elucidating the influence and mechanisms of maternal preeclampsia on human infants and suggest alerting the status of neurogenesis might be a promising approach to improve the long-term prognosis of offspring from preeclamptic pregnancy.
Acknowledgments The work was supported by grants from the National Science Fund of China $(81270712,81471470)$; the National Science Fund for Young Scholars of China $(81100438$, 81200448, 81200449, 81300506); Shanghai Municipal Natural Science Foundation (11ZR1404200, 12ZR1403700); Health industry special funds for Public Benefit Research Foundation from the Ministry of Health, People's Republic of China (201002013); the Science Fund of Shanghai, Program of Shanghai Subject Chief Scientist (12XD1401300); Program of Shanghai Leading Talent (2012); Shanghai Municipal Health Bureau (12GWZX0301); National Key Basic Research Plan of China (973 Plan) (2015CB943300); National Science Foundation for Young Scholars of Shanghai (13ZR1452000); Key Projects of Shanghai Municipal Health Bureau (20134031); Science Foundation for the Excellent Youth Scholars of Shanghai Municipal Health Bureau; the Shanghai Key Laboratory of Female Reproductive Endocrine-Related Diseases and the Key Specialty Project of the Ministry of Health, People's Republic of China; and Special Fund for Scientific Research in the Public Interest (201402006).

Conflict of Interest The authors declare that they have no conflict of interest.

Open Access This article is distributed under the terms of the Creative Commons Attribution License which permits any use, distribution, and reproduction in any medium, provided the original author(s) and the source are credited.

\section{References}

1. Griffith MI, Mann JR, McDermott S (2011) The risk of intellectual disability in children born to mothers with preeclampsia or eclampsia with partial mediation by low birth weight. Hypertens Pregnancy Off J Int Soc Study Hypertens Pregnancy 30(1):108-115. doi:10.3109/ 10641955.2010.507837

2. Gilbert WM, Danielsen B (2003) Pregnancy outcomes associated with intrauterine growth restriction. Am J Obstet Gynecol 188(6): 1596-1599, discussion 1599-1601

3. Barker DJ (2006) Adult consequences of fetal growth restriction. Clin Obstet Gynecol 49(2):270-283

4. Buhimschi I, Yallampalli C, Chwalisz K, Garfield RE (1995) Preeclampsia-like conditions produced by nitric oxide inhibition: effects of L-arginine, D-arginine and steroid hormones. Human Reprod (Oxf Engl) 10(10):2723-2730

5. Fantel AG, Nekahi N, Shepard TH, Cornel LM, Unis AS, Lemire RJ (1997) The teratogenicity of $N$ (omega)-nitro-L-ariginine methyl ester (L-NAME), a nitric oxide synthase inhibitor, in rats. Reprod Toxicol (Elmsford, NY) 11(5):709-717

6. Diaz V, Lebras-Isabet MN, Denjean A (2005) Effect of Nomeganitro-L-arginine methyl ester-induced intrauterine growth restriction on postnatal lung growth in rats. Pediatr Res 58(3):557-561. doi:10. 1203/01.pdr.0000179398.62365.43

7. Palmsten K, Buka SL, Michels KB (2010) Maternal pregnancyrelated hypertension and risk for hypertension in offspring later in life. Obstet Gynecol 116(4):858-864. doi:10.1097/AOG. 0b013e3181f3a1f9

8. Karumanchi SA, Maynard SE, Stillman IE, Epstein FH, Sukhatme VP (2005) Preeclampsia: a renal perspective. Kidney Int 67(6):21012113. doi:10.1111/j.1523-1755.2005.00316.x

9. Woodworth MB, Custo Greig L, Kriegstein AR, Macklis JD (2012) SnapShot: cortical development. Cell 151(4):918-918 e911. doi:10. 1016/j.cell.2012.10.004 
10. Pescosolido MF, Yang U, Sabbagh M, Morrow EM (2012) Lighting a path: genetic studies pinpoint neurodevelopmental mechanisms in autism and related disorders. Dialogues Clin Neurosci 14(3):239-252

11. Steegers EA, von Dadelszen P, Duvekot JJ, Pijnenborg R (2010) Preeclampsia. Lancet 376(9741):631-644. doi:10.1016/s0140-6736(10) 60279-6

12. Whitehouse AJ, Robinson M, Newnham JP, Pennell CE (2012) Do hypertensive diseases of pregnancy disrupt neurocognitive development in offspring? Paediatr Perinat Epidemiol 26(2):101-108. doi:10.1111/j. 1365-3016.2011.01257.x

13. Wu CS, Sun Y, Vestergaard M, Christensen J, Ness RB, Haggerty CL, Olsen J (2008) Preeclampsia and risk for epilepsy in offspring. Pediatrics 122(5):1072-1078. doi:10.1542/peds. 2007-3666

14. Vorhees CV, Williams MT (2006) Morris water maze: procedures for assessing spatial and related forms of learning and memory. Nat Protoc 1(2):848-858. doi:10.1038/nprot.2006.116

15. Lopez-Atalaya JP, Ciccarelli A, Viosca J, Valor LM, JimenezMinchan M, Canals S, Giustetto M, Barco A (2011) CBP is required for environmental enrichment-induced neurogenesis and cognitive enhancement. EMBO J 30(20):4287-4298. doi:10.1038/emboj. 2011.299

16. Ge WP, Miyawaki A, Gage FH, Jan YN, Jan LY (2012) Local generation of glia is a major astrocyte source in postnatal cortex. Nature 484(7394):376-380. doi:10.1038/nature10959

17. Stang1 D, Thuret $S$ (2009) Impact of diet on adult hippocampal neurogenesis. Genes Nutr 4(4):271-282. doi:10.1007/s12263-0090134-5

18. Ramel SE, Georgieff MK (2014) Preterm nutrition and the brain. World Rev Nutr Diet 110:190-200. doi:10.1159/000358467

19. Wullschleger S, Loewith R, Hall MN (2006) TOR signaling in growth and metabolism. Cell 124(3):471-484. doi:10.1016/j.cell. 2006.01.016

20. Hansen-Pupp I, Hellstrom-Westas L, Cilio CM, Andersson S, Fellman V, Ley D (2007) Inflammation at birth and the insulin-like growth factor system in very preterm infants. Acta Paediatr (Oslo, Norway 1992) 96(6):830-836. doi:10.1111/j.1651-2227.2007.00276.x

21. Kajantie E, Eriksson JG, Osmond C, Thornburg K, Barker DJ (2009) Pre-eclampsia is associated with increased risk of stroke in the adult offspring: the Helsinki birth cohort study. Stroke J Cereb Circ 40(4): 1176-1180. doi:10.1161/strokeaha.108.538025

22. Paulis L, Becker ST, Lucht K, Schwengel K, Slavic S, Kaschina E, Thone-Reineke C, Dahlof B, Baulmann J, Unger T, Steckelings UM (2012) Direct angiotensin II type 2 receptor stimulation in Nomeganitro-L-arginine-methyl ester-induced hypertension: the effect on pulse wave velocity and aortic remodeling. Hypertension 59(2): 485-492. doi:10.1161/hypertensionaha.111.185496

23. Freeman MR, Rowitch DH (2013) Evolving concepts of gliogenesis: a look way back and ahead to the next 25 years. Neuron 80(3):613623. doi:10.1016/j.neuron.2013.10.034

24. Martinez-Orgado J, Gonzalez R, Alonso MJ, Salaices M (2004) Impairment of fetal endothelium-dependent relaxation in a rat model of preeclampsia by chronic nitric oxide synthase inhibition. J Soc Gynecol Investig 11(2):82-88. doi:10.1016/j.jsgi.2003.08.003

25. Gereltsetseg G, Matsuzaki T, Iwasa T, Kinouchi R, Nakazawa H, Yamamoto S, Kuwahara A, Yasui T, Irahara M (2012) Delay in the onset of puberty of intrauterine growth retarded female rats cannot be rescued with hypernutrition after birth. Endocr J 59(11):963-972

26. Ming GL, Song H (2005) Adult neurogenesis in the mammalian central nervous system. Annu Rev Neurosci 28:223-250. doi:10. 1146/annurev.neuro.28.051804.101459
27. Jessberger S, Clark RE, Broadbent NJ, Clemenson GD Jr, Consiglio A, Lie DC, Squire LR, Gage FH (2009) Dentate gyrus-specific knockdown of adult neurogenesis impairs spatial and object recognition memory in adult rats. Learn Mem (Cold Spring Harb NY) 16(2): 147-154. doi:10.1101/lm.1172609

28. Campbell S, Macqueen G (2004) The role of the hippocampus in the pathophysiology of major depression. J Psychiatr Neurosci JPN 29(6):417-426

29. Rickmann M, Amaral DG, Cowan WM (1987) Organization of radial glial cells during the development of the rat dentate gyrus. J Comp Neurol 264(4):449-479. doi:10.1002/cne.902640403

30. Merkle FT, Tramontin AD, Garcia-Verdugo JM, Alvarez-Buylla A (2004) Radial glia give rise to adult neural stem cells in the subventricular zone. Proc Natl Acad Sci U S A 101(50):1752817532. doi:10.1073/pnas.0407893101

31. Li X, Barkho BZ, Luo Y, Smrt RD, Santistevan NJ, Liu C, Kuwabara T, Gage FH, Zhao X (2008) Epigenetic regulation of the stem cell mitogen FGF-2 by Mbd1 in adult neural stem/progenitor cells. J Biol Chem 283(41):27644-27652. doi:10.1074/jbc.M804899200

32. Waterhouse EG, An JJ, Orefice LL, Baydyuk M, Liao GY, Zheng K, $\mathrm{Lu} \mathrm{B}, \mathrm{Xu} \mathrm{B}$ (2012) BDNF promotes differentiation and maturation of adult-born neurons through GABAergic transmission. J Neurosci Off J Soc Neurosci 32(41):14318-14330. doi:10.1523/jneurosci. 070912.2012

33. Raballo R, Rhee J, Lyn-Cook R, Leckman JF, Schwartz ML, Vaccarino FM (2000) Basic fibroblast growth factor (FGF2) is necessary for cell proliferation and neurogenesis in the developing cerebral cortex. J Neurosci Off J Soc Neurosci 20(13):5012-5023

34. Stevens HE, Jiang GY, Schwartz ML, Vaccarino FM (2012) Learning and memory depend on fibroblast growth factor receptor 2 functioning in hippocampus. Biol Psychiatry 71(12):1090-1098. doi:10. 1016/j.biopsych.2012.03.013

35. Mudo G, Bonomo A, Di Liberto V, Frinchi M, Fuxe K, Belluardo N (2009) The FGF-2/FGFRs neurotrophic system promotes neurogenesis in the adult brain. J Neural Transm (Vienna Austria 1996) 116(8):9951005. doi:10.1007/s00702-009-0207-Z

36. Wang D, Song X, Wang Y, Li X, Jia S, Wang Z (2014) Gene expression profile analysis in epilepsy by using the partial least squares method. TheScientificWorldJOURNAL 2014:731091. doi: $10.1155 / 2014 / 731091$

37. Paradiso B, Marconi P, Zucchini S, Berto E, Binaschi A, Bozac A, Buzzi A, Mazzuferi M, Magri E, Navarro Mora G, Rodi D, Su T, Volpi I, Zanetti L, Marzola A, Manservigi R, Fabene PF, Simonato M (2009) Localized delivery of fibroblast growth factor-2 and brainderived neurotrophic factor reduces spontaneous seizures in an epilepsy model. Proc Natl Acad Sci U S A 106(17):7191-7196. doi:10. 1073/pnas.0810710106

38. Beaumont TL, Yao B, Shah A, Kapatos G, Loeb JA (2012) Layerspecific CREB target gene induction in human neocortical epilepsy. J Neurosci Off J Soc Neurosci 32(41):14389-14401. doi:10.1523/ jneurosci. 3408-12.2012

39. Covic M, Karaca E, Lie DC (2010) Epigenetic regulation of neurogenesis in the adult hippocampus. Heredity 105(1):122-134. doi:10.1038/hdy.2010.27

40. Ma DK, Jang MH, Guo JU, Kitabatake Y, Chang ML, Pow-Anpongkul N, Flavell RA, Lu B, Ming GL, Song H (2009) Neuronal activityinduced Gadd45b promotes epigenetic DNA demethylation and adult neurogenesis. Sci (New York, NY) 323(5917):1074-1077. doi:10. 1126/science.1166859 